\section{Ultrasonic Absorption in some Mixtures of an Unassociated and an Associated Liquid}

THe difference between classical and experimental values of ultrasonic absorption coefficients in unassociated liquids has generally been ascribed to relaxation phenomena in energy distribution among the internal degrees of freedom of the molecules.

Recently, J. M. M. Pinkerton ${ }^{1}$ and E. Bauer ${ }^{2}$ have presented theoretical interpretations of the absorption in a system (benzene - toluene) of two unassociated liquids with very different absorption coefficients. Their treatment assumes a behaviour strictly analogous to that of pure gases containing impurities. According to this interpretation, addition to an unassociated liquid of molecules of another liquid leads to a quicker attainment of the energy distribution. and hence decreases absorption.

We have studied mixtures composed of an unassociated liquid having large absorption and an associated liquid having much smaller absorption and low viscosity. In these associated liquids the origin of relaxation phenomena seems to be in the distortion of their structure produced by acoustic waves.

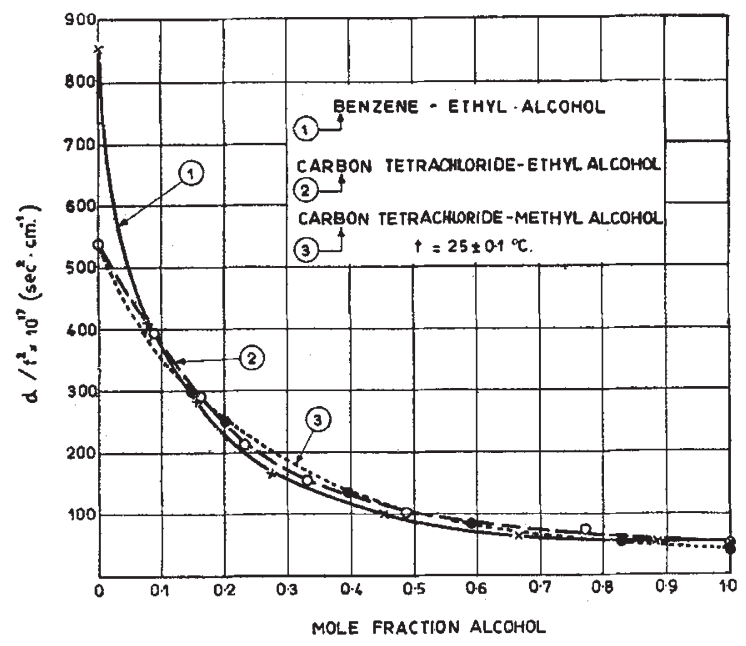

In the accompanying graph, the values of the parameter $\alpha / f^{2}$ ( $\alpha$ is amplitude absorption coefficient ; $f$ is frequency) for the systems benzene-ethyl alcohol, carbon tetrachloride - ethyl alcohol, carbon tetrachloride-methyl alcohol are given, measurements having been carried out by an optical method ${ }^{3}$, at $8 \mathrm{Mc} . \mathrm{s}$.

The curves are similar in every way to that for the system benzene - toluene. It is clear, therefore, that in the mixtures examined the variation of the absorption with concentration is chiefly due to the decrease of absorption in the unassociated component, which has a large coefficient, on account of the presence of different molecules, just as if the second component were also an unassociated liquid.

D. SETTE

Istituto Naz. Ultracustica,

Rome.

Feb. 2 .

${ }^{1}$ Pinkerton, J. M. M., Proc. Phys. Soc., B, 62, 129 (1949).

${ }^{2}$ Bauer, E., Proc. Phys. Soc., B, 62, 141 (1949).

${ }^{3}$ Sette, D., Nuovo Cim., 7, No. 1 (1950).

\section{Anharmonicity of the Lattice Oscillations in the Alkali Halide Crystals}

THE oscillations of the two interpenetrating lattices of the alkali and the halide ions respectively, in alkali halide crystals, with respect to each other, have been studied in detail, particularly in relation to the infra-red and the Raman spectra of the crystals, and the contribution of the electric dipole moments associated with these oscillations to the dielectric constants of the crystals. These oscillations are presumed to be anharmonic-the anharmonicity being attributed sometimes to the size of the amplitude of the oscillations-and the anharmonicity is invoked to explain the occurrence of certain combination frequencies in Raman spectra, and the temperature variation of the specific heat at constant volume at high temperatures. No attempt, however, seems to have been made to estimate the magnitude of the anharmonicity, though it can be done readily on the basis of any simple model of the crystal, as, for example, Born's, which regards the lattices of the positive and the negative ions respectively as held together by electrostatic forces between the ions, and by Van der Waals forces of repulsion between them, which die down much more rapidly with distance than the electrostatic forces. (The inclusion of forces due to the polarizabilities of the ions does not appreciably affect the results.)

Let us consider a halide of the $\mathrm{NaCl}$ type. Neglect. ing Van der Waals repulsions except between nearest neighbours, and taking the repulsion potential between two neighbouring ions to be $A \exp (-R / \rho)$, where $R$ is the distance between them, we obtain, for the potential energy of the crystal per ion,

$$
U=\frac{1}{2}\left\{-\alpha e^{2} / R+6 A \exp (-R / \rho)\right\},
$$

in which $\alpha=1.748$ is the Madelung constant. Now using the equilibrium condition $(\partial U / \partial R)_{R=d}=0$, where $d$ is the distance between the sodium and chloride ions, we may eliminate $A$ in the usual manner, and with the help of the relation $\left(\partial^{2} U / \partial R^{2}\right) R=d=9 d / \beta$, where $\beta$ is the isothermal compressibility, determine $p$. We thus find that, in sodium chloride, $d / \rho$, which we shall denote by $\delta$, is about 9 .

Now taking the cubic axes of the crystal as the $x y z$ axes of a co-ordinate system, and separating the $\mathrm{Na}^{+}$and the $\mathrm{Cl}^{-}$lattices from each other by a small distance $r$ in the direction $l m n$, we obtain for the potential energy of the deformed crystal per ion the expression

$U=U_{0}+a r^{2}+b r^{4}+c r^{4}\left(l^{4}+m^{4}+n^{4}\right)$,

in which

$$
\begin{aligned}
& U_{0}=-\frac{e^{2} \alpha}{2 d}\left(1-\frac{1}{\delta}\right)=-6 \cdot 4 \times 10^{-12} \\
& a=\frac{e^{2}}{d^{3}} \alpha \frac{(\delta-2)}{12}=1 \cdot 05 \times 10^{4} \\
& b=\frac{e^{2}}{d^{5}}\left\{\frac{21}{8}-\frac{7}{27 \sqrt{ } 3}-\alpha \frac{\left(2^{5}+3 \delta+3\right)}{48}\right\}= \\
& c=\frac{e^{2}}{d^{5}}\left\{-\frac{35}{8}+\frac{35}{81 \sqrt{ } 3}+\alpha \frac{\left(\delta^{3}+6 \delta^{2}+15 \delta+15\right)}{144}\right\} \\
& =1.62 \times 10^{20}
\end{aligned}
$$

In the expressions for $a, b$ and $c$, the terms involving $\alpha$ arise from Van der Waals repulsion, and the other terms in them from electrostatic interaction. In the numerical values, which refer to sodium chloride, the energies are expressed in ergs, and $r$ in $\mathrm{cm}$. 